\title{
Abundances Variations in PNe: Real or Illusory?
}

\author{
A. Riera $^{1,2}$, B. Balick ${ }^{3}$, G. Mellema ${ }^{4}$, K. Xilouris ${ }^{5}$ and Y. Terzian ${ }^{6}$ \\ ${ }^{1}$ Departament de Física i Enginyeria Nuclear, Universitat Politècnica de Catalunya; \\ ${ }^{2}$ Departament d'Astronomia i Meteorologia, Barcelona; \\ ${ }^{3}$ Department of Astronomy, University of Washington, Seattle; \\ ${ }^{4}$ Stockolm Observatory, Saltsjobaden; \\ ${ }^{5}$ National Astronomy and Ionosphere Observatory, Arecibo; \\ ${ }^{6}$ Space Sciences, Cornell University, Ithaca
}

In order to study the putative enrichment within a PN, we have undertaken deep longslit spectroscopic observations of selected targets in which we expected to find clear evidence of abundance gradients. The targets include NGC 3242: one of the PNs studied by Balick et al. (1994) in which they report N/H enhancements of $\sim 5$ in each FLIER; M1-18, M1-8, M3-3, NGC 6537 and Hubble 5. The observations were obtained at the 5-m Hale Telescope of the Palomar Observatory.

Temperatures were measured from the ratio of nebular and auroral forbidden lines of $\mathrm{N}^{+}$and $\mathrm{O}^{++}$in the usual manner. No small-scale temperature fluctuations were observed. Density structure was measured using the density-sensitive ratio of the $\mathrm{S}^{+}$forbidden lines at $\lambda 6717,6731 \AA$. With these physical properties mapped, it is straightforward to compute ionic abundance profiles. Total abundances were computed from the sum of all observed ionization species multiplied by an ionization correction factor ICF which accounts for unobserved ionization stages (Kingsburgh and Barlow 1994).

For the most part, the derived abundances in our sample fail to show the large changes reported in M1-75 by Guerrero et al (1995). On the other hand, in the most extreme case, NGC 3242, the standard methods yield factor-of-5 (or more) variations in N/H and S/H. Although the data are excellent, we question whether these $\mathrm{N} / \mathrm{H}$ and $\mathrm{S} / \mathrm{H}$ variations are real. Perhaps they result from unrealistic simplifying assumptions about cospatial ionization volumes used in the abundance measurement procedures. In any case, although N/H enhancements are plausible, it would be most surprising if the PN nucleus ejects sulfurenriched material.

The failure of the standard ICFs correction might be the result of charge exchange reactions, which decouple the ionization zones of the different low ionization species. In particular, $\mathrm{O}^{+}$might be converted to $\mathrm{O}^{\circ}$ in a region where $\mathrm{N}^{+}$and $\mathrm{S}^{+}$are the predominant ionization states. It is well known that the charge exchange reactions are especially important near the outer boundaries of a photoionized nebulae of moderate to high excitation, but numerical modelling is necessary to explore the relationship of the zone volumes of the different low ionization species.

\section{REFERENCES}

Balick, B., Perinotto, M., Maccioni, A., Terzian, Y. and Hajian, A., 1994, ApJ, 424, 800

Guererro, M., Stanghellini, L., and Manchado, A., 1995 ApJ, 444, L49-L52

Kingsburgh, R.L. and Barlow, M.J. 1994, MNRAS, 271, 257 